\title{
Consensus-based Distributed Approach to Lossy Economic Power Dispatch of Distributed Energy Resources
}

\author{
Xing, Hao ; Zeng, Pingliang; Mou, Yuting; Wu, Qiuwei
}

Published in:

International Transactions on Electrical Energy Systems

Link to article, DOI:

$10.1002 / 2050-7038.12041$

Publication date:

2019

Document Version

Peer reviewed version

Link back to DTU Orbit

Citation (APA):

Xing, H., Zeng, P., Mou, Y., \& Wu, Q. (2019). Consensus-based Distributed Approach to Lossy Economic Power Dispatch of Distributed Energy Resources. International Transactions on Electrical Energy Systems, 29(7), [e12041]. https://doi.org/10.1002/2050-7038.12041

\section{General rights}

Copyright and moral rights for the publications made accessible in the public portal are retained by the authors and/or other copyright owners and it is a condition of accessing publications that users recognise and abide by the legal requirements associated with these rights.

- Users may download and print one copy of any publication from the public portal for the purpose of private study or research.

- You may not further distribute the material or use it for any profit-making activity or commercial gain

- You may freely distribute the URL identifying the publication in the public portal 


\title{
Consensus-based Distributed Approach to Lossy Economic Power Dispatch of Distributed Energy Resources
}

\author{
Hao Xing ${ }^{\mathrm{a}}$, Pingliang Zeng ${ }^{\mathrm{a}, *}$, Yuting $\mathrm{Mou}^{\mathrm{b}}$, Qiuwei $\mathrm{Wu}^{\mathrm{a}, \mathrm{c}}$ \\ ${ }^{a}$ School of Automation, Hangzhou Dianzi University, Hangzhou, 310018 P. R. China \\ ${ }^{b}$ Center for Operations Research and Econometrics, Université catholique de Louvain, \\ Voie du Roman Pays 34, Louvain la Neuve, Belgium \\ ${ }^{c}$ Center for Electric Power and Energy (CEE), Department of Electrical Engineering, \\ Technical University of Denmark, Kgs. Lyngby, 2800 Denmark
}

\begin{abstract}
Economic dispatch problem (EDP) of distributed energy resources (DERs) is the key to achieve balanced coordination and economic operation of renewable energy and conventional generation, which is of vital significance to smart grids. This paper mainly investigates the EDP of DERs with transmission losses. We formulate the lossy EDP as an optimization problem, of which the objective is to minimize the aggregated production cost under physical constraints. It is a great challenge to solve the lossy EDP in a distributed fashion, as the non-convexity of the transmission loss formula has posed extra difficulties. This paper proposes a consensus-based distributed lambda iteration algorithm to solve the lossy EDP, and a damping mechanis$\mathrm{m}$ is designed to overcome the oscillatory problem that conventional lambda iterations often suffer. The proposed algorithm is further improved, in order to tackle the prohibited operating zones of generators. Exhaustive simulations are presented to illustrate the proposed algorithm.
\end{abstract}

Keywords: Distributed algorithm, economic dispatch, smart grid,transmission losses, prohibited operating zones.

\footnotetext{
${ }^{*}$ Corresponding author

Email addresses: xing.h@hdu.edu.cn (Hao Xing), plz@hdu.edu.cn (Pingliang Zeng), yuting.mou@uclouvain.be (Yuting Mou), qw@elektro.dtu.dk (Qiuwei Wu)
} 


\section{Introduction}

At the beginning of 1990s, power companies were faced with a huge growth of electrical energy consumption due to the increase of population and electrification, which challenged centralized supply because of the inability of transmission lines and energy losses [1]. In this background, distributed energy resources were put on the table again. In the late 1990s, electricity from wind and solar became commercially feasible. Nowadays, many countries have ambitious target of renewable penetration. Meanwhile, advanced information communication technology makes smart grids possible. With the rapidly growing penetration of renewable generation and fast development of smart grid technologies, modern power system is evolving towards the direction where distributed energy resources are becoming increasingly prevalent. In this new paradigm of distributed energy resources (DERs), electricity production from solar and wind is likely to be consumed locally and in each local area, conventional generators are installed to help the main grid maintain stability. These conventional generators are connected within a local area, as well as interconnecting with other areas. In order to better exploit these generators, an coordination scheme is desirable to dispatch them economically.

Many centralized algorithms based on linear programming and quadratic programming have been proposed to solve the economic dispatch problem (EDP). Nevertheless, researchers are increasingly interested in distributed algorithms owning to the trend of smart grids [2]. Future smart grid is likely a large scale system [3], incorporating numerous DERs. The widely spatial distribution of power generation systems adds extra difficulties in solving the EDP. Recently, many distributed algorithms to deal with large scale systems, have been put forward, e.g., $[4,5]$. Compared with centralized algorithms, distributed algorithms exhibit many benefits including scalability , reinforced robustness, and evenly-allocated computation and communication burdens $[6,7]$.

In recent years, numerous distributed algorithms have been presented to address the EDP in smart grid under the assumption of an ideal model. In [8] and [9], the authors propose the incremental cost consensus (ICC) algorithm to solve the EDP, in which, the supply and demand balance is guaranteed using average consensus. A consensus + innovation approach is put forward in [10]. This approach ensures the commonly shared optimal incremental cost by using consensus while the demand and supply balance is guaran- 
teed by the innovation term. In [11], the authors propose a consensus based distributed algorithm, which enables the generators to collectively learn the mismatch between demand and total supply for feedback. Two fully distributed algorithms for the EDP are also proposed in our previous works [12] and [13], respectively. The algorithm proposed in [12] deals with the EDP with quadratic cost functions on connected undirected graphs, and then is extended to deal with the EDP with general convex functions on strongly connected directed graphs in [13].

The major deficiency of these aforementioned algorithms is that many practical constraints are ignored including transmission losses and prohibited operating zones [8]-[14]. The negligence of these practical constraints may lead to demand and supply imbalance and endanger stable operation of generators. Those practical constraints complicate the EDP. Specifically, transmission losses bring nonlinearity to the model, whereas prohibited operating zones makes the feasible region discontinuous, both rendering the EDP non-convex [15]. To solve the EDP with transmission losses, the conventional lambda-iteration method has been widely used in the power industry where the total transmission losses are approximated by the $\mathbf{B}$ matrix loss formula [16]. The conventional lambda-iteration method, which is in essence an implicit form of the fixed-point iteration method, solves the non-convex EDP by solving an approximated convex problem in each iteration. Nevertheless, the conventional lambda-iteration method has two noticeable shortcomings:

1) As widely reported in the literature, e.g., [17], [18], the conventional lambda-iteration method may exhibit the oscillatory behavior in large-scale mixed generation systems, which reduces the computational efficiency. According to [19], it is common practice that the power assignment with the minimum generation cost among the oscillatory solutions is chosen as the optimal one. However, we note that the "optimal" solution in that case is not even feasible, because for such iterative algorithms based on Lagrangian duality theory, demand and supply is balanced only when the Lagrangian multiplier converges to an unique value.

2) In [17], it is pointed out that the conventional lambda-iteration method cannot directly deal with the discontinuity incurred by the prohibited operating zones. Although some heuristics for searching a feasible solution in the neighbourhood of the optimal solutions have been proposed 
in [20] and [21], there lack theoretical proof for guaranteed convergence to the optimal solution.

Continuing along the trend of distributed algorithms and the necessity of including more practical constraints in the EDP, in this paper we aim at solving the EDP with transmission losses and prohibited operating zones in a distributed fashion and propose the distributed augmented lambda-iteration algorithm. The challenges for this work mainly stem from the non-convexity of the EDP and the restriction of distributed algorithmic architecture. Compared with the conventional lambda-iteration method, the proposed algorith$\mathrm{m}$ in this paper is augmented with the following features:

1) A damping mechanism is designed to avoid the oscillatory problem that the conventional lambda-iteration method usually suffers from.

2) The proposed algorithm is improved by using Pseudo marginal costs to tackle the discontinuity due to prohibited operating zones.

3) The proposed algorithm is applicable for general convex cost functions and is not restricted to quadratic costs, which is assumed by most existing works.

Furthermore, the proposed algorithm is distributed in the sense that it does not rely on any pre-assigned central/leader node. Based on the average consensus algorithm [22], in our algorithm all the nodes conduct local computation and communicate with their neighbors in a connected undirected network to solve the EDP. Although many works also attack the EDP in the context of distributed algorithms, they only assume the ideal convex ED$\mathrm{P}$ model which is not subject to the non-convexity caused by transmission losses and prohibited operating zones. Therefore, the distributed algorithms proposed by [8]-[23] are unapplicable to the non-convex EDP. In contrast, the proposed distributed algorithm has the advantage of solving the EDP with transmission losses and prohibited operating zones. Reference [19] also takes into consideration transmission losses and proposes a distributed consensus-based algorithm. However, the authors neglect the penalty factors of each generator resulting in the convergences to a feasible solution rather than the optimal one. In [24], a multi-stage decentralized algorithm based on the flooding protocol and differential evolution algorithm (DEA) is proposed, where the flooding protocol is used to broadcast messages and each node solves a local optimization problem using the DEA. The control 
parameters of the DEA are very sensitive to the problem's dimension and their algorithm lacks the self-tuning feature, which limits the applicability. By contrast, the alforithm proposed in this study is free from this issue.

The remaining parts of the paper are organized as follows. We present the problem formulation of lossy EDP in Section 2. The conventional lambdaiteration method as well as the average consensus algorithm is introduced in Section 3. We propose the consensus based distributed algorithm for lossy EDP in Section 4. Extensive numerical results are given in Section 5 to show the performance of the proposed algorithm. Finally, we conclude our paper in Section 6.

\section{Problem Formulation}

In this section, the problem formulation of the lossy EDP is presented.

Suppose that there are totally $n$ generators in the power grid, labelled from 1 to $n$. Let us denote the total load and output of the $i$ th generator by $P^{\text {load }}$ and $P_{i}$, respectively. Taking into consideration the transmission losses and prohibited operation zones of generators, we formulate the EDP as follows:

- Objective:

$$
\min \sum_{i=1}^{n} F_{i}\left(P_{i}\right),
$$

where $F_{i}\left(P_{i}\right)$ is the cost function associated with the $i$ th generator.

- Power balance constraint:

$$
\sum_{i=1}^{n} P_{i}-P^{\text {loss }}-P^{\text {load }}=0
$$

where $P^{\text {loss }}$ is the total transmission losses over the power grid.

- Capacity constraints of generators:

$$
\underline{P}_{i} \leqslant P_{i} \leqslant \bar{P}_{i}, \forall i=1, \ldots, n
$$

where $\underline{P}_{i}$ and $\bar{P}_{i}$ are the lower bound and upper bound of the output of the $i$ th generator, respectively. 
In most cases, the cost functions are assumed to be in the following quadratic form:

$$
F_{i}\left(P_{i}\right)=\frac{1}{2} \alpha_{i} P_{i}^{2}+\beta_{i} P_{i}+\gamma_{i}
$$

where $\alpha_{i}>0, \beta_{i}$, and $\gamma_{i}$ are cost parameters. In this paper, we deal with the EDP with general cost functions satisfying the assumption below:

Assumption 1 : For every $1 \leqslant i \leqslant n, F_{i}\left(P_{i}\right): \mathbb{R}_{+} \rightarrow \mathbb{R}_{+}$is strictly convex and twice continuously differentiable with

$$
\frac{d f_{i}\left(P_{i}\right)}{d P_{i}} \geqslant 0, \quad \forall P_{i} \in \mathbb{R}_{+},
$$

where $f_{i}=\frac{d F_{i}\left(P_{i}\right)}{d P_{i}}$ is the first derivative of function $F_{i}\left(P_{i}\right), \mathbb{R}_{+}$denotes the set of nonnegative real numbers, and the equality holds at isolated points only. It can be easily verified that the quadratic function (4) satisfies the assumption 1 .

We assume that the total transmission losses are a function of the generator outputs $P_{i}$ 's and we use the $\mathbf{B}$ matrix loss formula to represent $P^{\text {loss }}$, given by

$$
P^{\text {loss }}=\sum_{i=1}^{n} \sum_{j=1}^{n} P_{i} B_{i j} P_{j}+\sum_{i=1}^{n} B_{0 i} P_{i}+B_{00},
$$

where $B_{i j}=B_{j i}, B_{0 i}$, and $B_{00}$ are computed according to the line parameters and the average daily operating status of the power systems [25, Chap. 13.3]. The equality constraint (2) contains a quadratic term due to the loss formula, which also leads to the non-convexity of the EDP (1)-(3).

Remark 1. Although total transmission losses can also be computed using the power flow equations, we use the $\mathbf{B}$ matrix loss formula, for it can give a sufficiently accurate estimation of the total transmission losses in the off-line mode with a small amount of computation [15].

Remark 2. In the proposed optimization model (1)-(3), the modelling of DERs is achieved through the cost function and the physical constraints associated with each DER. Firstly, each DER is associated with a local cost function $F_{i}\left(P_{i}\right)$, with their sum $\sum_{i=1}^{n} F_{i}\left(P_{i}\right)$ being the operating cost of the whole system. Secondly, the inequality constraints of the proposed model are the union of the local capacity constraints of each DER. Furthermore, the number of DERs is in accordance with the number of cost terms in the objective function, as well as the the number of capacity constraints (3). Therefore, 
the proposed problem formulation is generalizable to DER population of any size.

For the implementation of the proposed distributed algorithm, we construct a communication network on the power grid with each node associated with a bus in the power grid. Two connected undirected graphs with selfloops are established in this paper: one consists of pure generation bus and the other one consists of $m$ buses associated with pure load, pure generation, or both. We construct two graphs denoted by $G=(V, E)$ and $G^{\prime}=\left(V^{\prime}, E^{\prime}\right)$, respectively. The node set $V^{\prime}$ consists of all the $m$ buses in the grid, while $V$ consists of all the $n$ generation buses, i.e., $V^{\prime}=\{1,2, \ldots, n, n+1, \ldots, m\}$ and $V=\{1,2, \ldots, n\}$. We also assume that $G$ and $G^{\prime}$ are sparse graphs in the sense that

$$
\max _{1 \leqslant j \leqslant m} d_{j}^{\prime} \ll m, \quad \max _{1 \leqslant i \leqslant n} d_{i} \ll n
$$

\section{Introduction to Conventional Lambda-iteration Method}

In this section we introduce the conventional lambda-iteration method [15], which was originally designed for EDP with quadratic cost functions and transmission losses.

The Lagrange function of the EDP (1)-(3) is given by

$$
L=\sum_{i=1}^{n} F_{i}\left(P_{i}\right)-\lambda\left(\sum_{i=1}^{n} P_{i}-P^{\text {loss }}-P^{\text {load }}\right),
$$

where $\lambda$ is the Lagrange multiplier.

The optimal solution and the optimal Lagrange multiplier, denoted by $P_{i}^{*}$ and $\lambda^{*}$ respectively, satisfy

$$
\frac{\partial L}{\partial P_{i}^{*}}=f_{i}\left(P_{i}^{*}\right)-\lambda^{*}\left(1-\frac{\partial P^{\text {loss }}}{\partial P_{i}^{*}}\right)=0, \forall i,
$$

where $f_{i}(r)=\alpha_{i} r+\beta_{i}$. Combining the cost functions (1) and the inequality constraints (3) yields the optimality condition that for all $i \in V$,

$$
\begin{cases}f_{i}\left(P_{i}^{*}\right) p f_{i}>\lambda^{*}, & \text { for } P_{i}^{*}=\underline{P}_{i}, \\ f_{i}\left(P_{i}^{*}\right) p f_{i}=\lambda^{*}, & \text { for } \underline{P}_{i}<P_{i}^{*}<\bar{P}_{i}, \\ f_{i}\left(P_{i}^{*}\right) p f_{i}<\lambda^{*}, & \text { for } P_{i}^{*}=\bar{P}_{i},\end{cases}
$$


where

$$
p f_{i}=1 /\left(1-\frac{\partial P^{\text {loss }}}{\partial P_{i}}\right)
$$

is the penalty factor. From the loss formula (5), it follows that

$$
p f_{i}=1 /\left(1-2 \sum_{j=1}^{n} B_{i j} P_{j}-B_{0 i}\right) .
$$

We can obtain the optimal solution $P_{i}^{*}$ 's and the optimal Lagrange multiplier $\lambda^{*}$ by combining (2) and (7). But the equality constraint (2) has a quadratic term incurred by the loss formula (5), and the inequation (7) is non-linearly piecewise, therefore it is difficult to solve (2) and (7) directly. We now introduce the conventional lambda-iteration method [15], which can solve (2) and (7) iteratively. Denote the iteration step denoted by $k=0,1, \ldots$

Step 1: At step $k=0$, the generators pick initial values $P_{i}[0]$ 's such that $\sum_{i=1}^{n} P_{i}[0]-P^{\text {load }}=0$.

Step 2: Compute the penalty factors $p f_{i}[k]$ 's and the total transmission losses $P^{\text {loss }}[k]$ according to (8) and (5), respectively.

Step 3: Solve the following equations to get $P_{i}[k+1]$ 's and $\lambda[k+1]$.

$$
\begin{gathered}
\sum_{i=1}^{n} P_{i}[k+1]-P^{\text {loss }}[k]-P^{\text {load }}=0, \\
\begin{cases}f_{i}\left(P_{i}[k+1]\right) p f_{i}[k]>\lambda[k+1], & P_{i}[k+1]=\underline{P}_{i}, \\
f_{i}\left(P_{i}[k+1]\right) p f_{i}[k]=\lambda[k+1], & P_{i}[k+1] \in\left(\underline{P}_{i}, \bar{P}_{i}\right), \\
f_{i}\left(P_{i}[k+1]\right) p f_{i}[k]<\lambda[k+1], & P_{i}[k+1]=\bar{P}_{i} .\end{cases}
\end{gathered}
$$

Step 4: Go back to Step 2 and loop until convergence.

The core idea of the lambda-iteration method is to use the penalty factors and the transmission losses derived from the result of the previous iteration to solve the next iteration. Then the $P^{l o s s}[k]$ and $p f_{i}[k]$ 's in (9) and (10) are treated as known parameters rather than variables. One can readily solve (9) and (10) using convex optimization algorithms, e.g., gradient-based algorithms, which is much easier than directly solving (2) and (7).

\section{Distributed Lambda-iteration Algorithm}

In this section we propose the consensus-based distributed lambda-iteration method. 


\subsection{Distributed Collection of Demand Information}

The lossy EDP formulation is based on an implicit assumption that the aggregate demand information is known to each generator. Nevertheless, in practical power grids the demand is spatially distributed at almost all the buses, i.e., $P^{\text {load }}=\sum_{j=1}^{m} P_{j}^{\text {load }}$, where $P_{j}^{\text {load }}$ is the power demand at bus $j$. As pointed out in [13], it is unnecessary for the generators to know the aggregated demand $P^{l o a d}$. Instead, we apply the average consensus algorithm [22] so that each node in $V$ gets the average demand $\eta^{*}=P^{l o a d} / n$, which as we will show, is sufficient to solve the lossy EDP.

In graph $G^{\prime}=\left(V^{\prime}, E^{\prime}\right)$, define an associated doubly stochastic matrix $Q^{\prime} \in \mathbb{R}^{m \times m}$ using the Metropolis weights [22]. For every node $i \in V^{\prime}$, we establish two variables $p_{i}(\kappa)$ and $s_{i}(\kappa)$, respectively initialized by $p_{i}(0)=$ $P_{i}^{\text {load }}$, and

$$
s_{i}(0)= \begin{cases}1, & i=1,2, \ldots, n, \\ 0, & i=n+1, n+2, \ldots m .\end{cases}
$$

Note that initialization of $s_{i}(\kappa)$ is in a heterogenous fashion. We then run the following average consensus algorithms simultaneously until convergence:

$$
\begin{aligned}
& p_{i}(\kappa+1)=q_{i i}^{\prime} p_{i}(\kappa)+\sum_{j \in N_{i}^{\prime}} q_{i j}^{\prime} p_{j}(\kappa), \\
& s_{i}(\kappa+1)=q_{i i}^{\prime} s_{i}(\kappa)+\sum_{j \in N_{i}^{\prime}} q_{i j}^{\prime} s_{j}(\kappa) .
\end{aligned}
$$

Defining $p^{*}=\lim _{\kappa \rightarrow \infty} p_{i}(\kappa)$ and $s^{*}=\lim _{\kappa \rightarrow \infty} s_{i}(\kappa)$, we have:

$$
p^{*}=P^{\text {load }} / m, s^{*}=n / m .
$$

For every node $i \in V$, we have:

$$
\eta^{*}=\frac{p^{*}}{s^{*}}=P^{l o a d} / n
$$

\subsection{Distributed Determination of $P_{i}[0]$ 's}

This subsection aims at the determination of $P_{i}[0]$ 's in a distributed fashion, satisfying the inequality constraints (3) and 


$$
\sum_{i=1}^{n} P_{i}[0]=P^{\text {load }}+B_{00}=n\left(\eta^{*}+B_{00} / n\right) .
$$

Note that we extract the term $B_{00}$ from the loss formula and put it in the above equation, for $B_{00}$ is a constant and $B_{00} / n$ is assumed to be known by each generator. The average consensus algorithm is also used here in order that each node gets the estimation of the aggregate generation capability of all the generators.

Consequently, each node $i \in V$ establishes two auxiliary variables $\underline{x}_{i}(\kappa)$ and $\bar{x}_{i}(\kappa)$, initialized by $\underline{x}_{i}(0)=\underline{P}_{i}, \bar{x}_{i}(0)=\bar{P}_{i}$. Then run the following average consensus iterations till convergence,

$$
\begin{aligned}
& \underline{x}_{i}(\kappa+1)=q_{i i} \underline{x}_{i}(\kappa)+\sum_{j \in N_{i}} q_{i j} \underline{x}_{j}(\kappa), \forall i \in V, \\
& \bar{x}_{i}(\kappa+1)=q_{i i} \bar{x}_{i}(\kappa)+\sum_{j \in N_{i}} q_{i j} \bar{x}_{j}(\kappa), \forall i \in V,
\end{aligned}
$$

When iterations (14) and (15) converge, each node $i \in V$ will get the common values $\underline{x}^{*}$ and $\bar{x}^{*}$ similarly given by

$$
\underline{x}^{*}=\left(\sum_{i=1}^{n} \underline{P}_{i}\right) / n, \bar{x}^{*}=\left(\sum_{i=1}^{n} \bar{P}_{i}\right) / n .
$$

After obtaining $\underline{x}^{*}$ and $\bar{x}^{*}$, the nodes can get the $P_{i}[0]$ 's following

$$
P_{i}[0]=\underline{P}_{i}+\frac{x^{*}-\underline{x}^{*}}{\bar{x}^{*}-\underline{x}^{*}}\left(\bar{P}_{i}-\underline{P}_{i}\right), \forall i \in V,
$$

where $x^{*}=\eta^{*}+B_{00} / n$.

\subsection{Distributed Computation of $p f_{i}[k]$ 's and $P_{a}^{\text {loss }}[k]$}

We assume that each node $i$ knows the $\mathbf{B}$ coefficients associated with itself, i.e., $B_{i j}, \forall j \in V$. Hence, the key to calculating $p f_{i}[k]$ is to calculate $\sum_{j=1}^{n} B_{i j} P_{j}[k]$ in a distributed fashion. For this purpose, each node $i \in V$ establishes an auxiliary variable $y_{i}^{j}(\kappa)$, where the superscript $j$ represents $y_{i}^{j}$ is meant for the calculation of $p f_{j}[k]$. We initialize $y_{i}^{j}$ 's according to

$$
y_{i}^{j}(0)=B_{i j} P_{i}[k]
$$


Then run the following iteration till convergence,

$$
y_{i}^{j}(\kappa+1)=q_{i i} y_{i}^{j}(\kappa)+\sum_{l \in N_{i}} q_{i l} y_{l}^{j}(\kappa), \forall i \in V .
$$

Define $y^{j *}=\left(\sum_{i=1}^{n} B_{i j} P_{i}[k]\right) / n$, then the penalty factor $p f_{j}$ is given by

$$
p f_{j}[k]=1 /\left(1-2 n y^{j *}-B_{0 j}\right) .
$$

Loop until all the nodes $j \in V$ obtains their $p f_{j}[k]$ 's.

We then compute the total transmission losses $P^{\text {loss }}[k]$. Since the constant term $B_{00}$ has already been included in $x^{*}$, we only need to compute

$$
\begin{aligned}
P_{a}^{\text {loss }}[k] & =P^{\text {loss }}[k]-B_{00} \\
& =\sum_{i=1}^{n} \sum_{j=1}^{n} P_{i}[k] B_{i j} P_{j}[k]+\sum_{i=1}^{n} B_{0 i} P_{i}[k] \\
& =\sum_{i=1}^{n}\left(n y^{i *}[k] P_{i}[k]+B_{0 i} P_{i}[k]\right) .
\end{aligned}
$$

For this purpose, each node establishes an auxiliary variable $y_{i}(\kappa)$, initialized by

$$
y_{i}(0)=n y^{i *}[k] P_{i}[k]+B_{0 i} P_{i}[k] .
$$

And then run the following average consensus algorithm till convergence,

$$
y_{i}(\kappa+1)=q_{i i} y_{i}(\kappa)+\sum_{j \in N_{i}} q_{i j} y_{j}(\kappa), \forall i \in V
$$

Denote the convergence value of $(23)$ by $y^{*}$, it follows that

$$
y^{*}=\sum_{i=1}^{n}\left(n y^{i *}[k] P_{i}[k]+B_{0 i} P_{i}[k]\right) / n=P_{a}^{\text {loss }}[k] / n .
$$

\subsection{Distributed Bisection Algorithm for $P_{i}[k+1]$ 's and $\lambda[k+1]$}

With the $p f_{i}[k]$ 's and $P_{a}^{\text {loss }}[k]$ obtained in a distributed fashion, we now proceed to the updates of $P_{i}[k+1]$ 's and $\lambda[k+1]$, which corresponds to step 3 of the conventional lambda-iteration method. 
The updating of $P_{i}[k+1]$ 's and $\lambda[k+1]$ is equivalent to solving the following optimization problem:

$$
\begin{array}{ll}
\min & \sum_{i=1}^{n} p f_{i}[k] F_{i}\left(P_{i}\right) \\
\text { s.t. } & P_{i} \in \Omega_{i}, \forall i \in V, \\
& \sum_{i=1}^{n} P_{i}-P^{\text {loss }}[k]-P^{\text {load }}=0,
\end{array}
$$

where for all i, $\Omega_{i}$ is defined as the set of real numbers that satisfies the local inequality constraint. One can verify that the equations (9) and (10) which are solved in step 3 of the conventional lambda-iteration method, are the necessary optimality condition of problem (25)-(27) with constraints (26) replaced by (3).

In our previous work [13], the distributed bisection algorithm is proposed for the EDP in the form of (25). Let us define the following mapping:

$$
\mathcal{P}_{i}(\lambda)= \begin{cases}\underline{P}_{i}, & h_{i}(\lambda)<\underline{P}_{i}, \\ h_{i}(\lambda), & h_{i}(\lambda) \in \Omega_{i}, \\ \bar{P}_{i}, & h_{i}(\lambda)>\bar{P}_{i}\end{cases}
$$

where $h_{i}(\cdot)=g_{i}\left(\frac{\lambda}{p f_{i}[k]}\right)$ and $g_{i}(\cdot)$ is the inverse function of $f_{i}(\cdot)$. One can verify that the mapping (28) is monotonically increasing with respect to $\lambda$. So $P_{i}[k+1]=\mathcal{P}_{i}(\lambda[k+1])$ is also monotonically increasing with respect to $\lambda[k+1]$, which allows us to adopt the idea of bisection. The detailed procedures are as follows.

Each node establishes two commonly shared variables $\lambda^{+}$and $\lambda^{-}$such that the optimal Lagrange multiplier must lie in the interval $\left[\lambda^{-}, \lambda^{+}\right]$. The initial $\lambda^{+}$and $\lambda^{-}$can be selected to be extremely large and small, respectively, for the convergence of bisection is very fast.

Let $t=0,1, \ldots$ denote the bisection steps. At step $t$, each node computes $\lambda(t+1)=\left(\lambda^{-}(t)+\lambda^{+}(t)\right) / 2$.

Each node then obtains $P_{i}(\lambda(t+1))$ according to (28) with $\lambda[k+1]$ replaced by $\lambda(t+1)$, and then establishes a variable $z_{i}(\kappa)$, which is initialized by $z_{i}(0)=P_{i}(\lambda(t+1))$. Run the following iteration till convergence,

$$
z_{i}(\kappa+1)=q_{i i} z_{i}(\kappa)+\sum_{j \in N_{i}} q_{i j} z_{j}(\kappa), \forall i \in V .
$$


After convergence, denote the convergence value by $z^{*}$. Then each node updates $\lambda^{-}(t+1)$ and $\lambda^{+}(t+1)$ according to

- For $z^{*}<y^{*}[k]+x^{*}, \lambda^{+}(t+1)=\lambda^{+}(t), \lambda^{-}(t+1)=\lambda(t+1)$.

- For $z^{*}=y^{*}[k]+x^{*}, \lambda[k+1]=\lambda(t+1)$, and the bisection stops.

- For $z^{*}>y^{*}[k]+x^{*}, \lambda^{+}(t+1)=\lambda(t+1), \lambda^{-}(t+1)=\lambda^{-}(t)$.

Recompute $P_{i}(\lambda(t+1))$ 's and circulate the bisection until convergence. Then each node obtains $P_{i}[k+1]$ and $\lambda[k+1]$.

Remark 3. The above results can be understood from an economic perspective. The given $\lambda$ is the marginal cost of a power injection at a slack bus (if there is one), while $\lambda / p f_{i}[k]$ is the local marginal price (LMP) at the ith generator, which is distorted from $\lambda$ by the penalty factor $p f_{i}[k]$. Let us consider the problem from a Lagrange dual perspective. The Lagrange function of the problem (25)-(27) is given by

$$
L=\sum_{i=1}^{n} p f_{i}[k] F_{i}\left(P_{i}\right)-\lambda\left(\sum_{i=1}^{n} P_{i}-P^{\text {loss }}[k]-P^{\text {load }}\right) .
$$

For a given Lagrange multiplier $\lambda$, consider the following optimization problem:

$$
\begin{array}{ll}
\min & L, \\
\text { s.t. } & P_{i} \in \Omega_{i}, \forall i \in V .
\end{array}
$$

Since the Lagrange multiplier $\lambda$ is given, the objective of (31) is equivalent to

$$
\min L^{o}=\sum_{i=1}^{n} p f_{i}[k] F_{i}\left(P_{i}\right)-\lambda \sum_{i=1}^{n} P_{i} .
$$

Note that in the optimization problem (31), the $P_{i}$ 's are mutually independent, for the equality constraint is null (reflected indirectly in $\lambda$ ). With $p f_{i}[k]>0$, the problem (31) can be equivalently divided into $n$ subproblems:

$$
\begin{array}{ll}
\min & L_{i}^{o}=F_{i}\left(P_{i}\right)-\frac{\lambda P_{i}}{p f_{i}[k]}, \\
\text { s.t. } & P_{i} \in \Omega_{i},
\end{array}
$$

The function $-L_{i}^{o}=\frac{\lambda P_{i}}{p f_{i}[k]}-F_{i}\left(P_{i}\right)$ is the net revenue of the ith generator. Therefore the objective of the problem (33) is to maximize the net revenue of each generator, given a fixed LMP. 
Remark 4. In general, this paper is mainly about investigating a completely new and different algorithmic paradigm for a problem that has been solved in traditional frameworks, i.e., the fully distributed approach to the lossy economic dispatch problem. Since the result analysis of lossy EDP itself can be easily found in literature [15], the key issue here is, whether the proposed distributed approach has the same optimality as the traditional centralized methods. Through the theoretical results in Section 4 and the numerical experiments to be presented in Section 6, it can be easily verified that the proposed distributed approach, although only using local communication and distributed computation, can still achieve exactly the same optimal result that traditional methods obtain based on global information and central computation.

\section{Algorithm Improvements}

In this section, we first propose a damping mechanism to address the oscillatory problem in the lambda iterations, and then extended the proposed algorithm to the case with prohibited operating zones.

\subsection{Damping Mechanism to Avoid Oscillations}

As aforementioned, the conventional lambda-iteration method may exhibit oscillatory behavior. That is, after a certain amount of iterations, the results of each lambda-iteration oscillate periodically between 2 (hardly more than 2) values, instead of converging to a unique solution. To the best of the authors' knowledge, none of the existing works have effectively solved this issue. In this subsection, we propose a damping mechanism, which can effectively avoid such oscillation and make the iterations converge to the unique optimal solution.

Here we assume that after enough iteration steps, the results oscillate between two values, i.e., for some integer $K>0$ and $l=1,2, \ldots, P[k]=P[k+$ $2 l]$, and $P[k] \neq P[k+1], \forall k>K$, where $P[k]=\left[P_{1}[k], \ldots, P_{n}[k]\right]^{T} \in \mathbb{R}^{n}$. We note that both in subsection IV-C and in the step 2 of the conventional lambda-iteration method, the computation of $p f_{i}[k]$ 's and $P_{a}^{\text {loss }}[k]$ only depends on $P_{i}[k]$ 's, without using the results of previous iterations. Define the following damping operator

$$
D[k]=\frac{(P[k]+P[k-1])}{2},
$$


which takes the average of the computational results at iterations $k$ and $k-1$. We then use $D[k]$ instead of $P[k]$ to compute $p f_{i}[k]$ 's and $P_{a}^{\text {loss }}[k]$. We have the following two propositions:

Proposition 1. The damping operator $D[k]$ prevents the lambda-iteration from oscillating between 2 values.

Proof: Since the lambda-iteration method is an implicit form of the fixedpoint iteration method, it can be expressed by $P[k+1]=H(P[k])$, where $H(\cdot)$ is the implicit function corresponding to the lambda-iteration without $D[k]$. Therefore the lambda-iteration with $D[k]$ is given by $P[k+1]=H((P[k]+$ $P[k-1]) / 2)$.

We then prove Proposition 1 by contradiction. Assume that after enough iteration steps, the lambda-iteration with $D[k]$ oscillates between $u$ and $v$. Therefore we have for some $k$,

$$
P[k]=u, P[k+1]=v, P[k+2]=u, P[k+3]=v,
$$

where $u \neq v$. Note that

$$
\begin{gathered}
P[k+2]=H\left(\frac{P[k+1]+P[k]}{2}\right)=H\left(\frac{v+u}{2}\right), \\
P[k+3]=H\left(\frac{P[k+2]+P[k+1]}{2}\right)=H\left(\frac{u+v}{2}\right) .
\end{gathered}
$$

It follows that $P[k+3]=P[k+2]$, which contradicts the assumption that $u \neq v$. Therefore Proposition 1 is proved.

Proposition 2. The damping operator $D[k]$ does not distort the convergence value from the optimal solution.

Proof: The optimal solution of the EDP satisfies $P^{*}=H\left(P^{*}\right)$. Let us consider the lambda-iteration with the damping operator $D[k]$. When converged, the convergence value, denoted by $P^{\prime}$, satisfies $P^{\prime}=H\left(\left(P^{\prime}+P^{\prime}\right) / 2\right)=H\left(P^{\prime}\right)$. Therefore we have $P^{\prime}=P^{*}$.

In practice, there is a very small chance that the lambda-iterations oscillate between $l>2$ values. For instance, as shown in our case studies, a test on 15-unit bus system shows oscillation between three solutions. To further deal with this, the damping operator can be generalized in the following manner:

$$
D[k]=\frac{P[k]+\ldots+P[k-l+1]}{l} .
$$




\subsection{Dealing with Prohibited Operating Zones}

For each generator $i \in V$, suppose that there are in total $M_{i}$ prohibited operating zones, and define $\underline{P}_{i}^{j}$ and $\bar{P}_{i}^{j}$ as the lower and upper bounds of the $j$ th prohibited operating zone, respectively. Therefore the permitted operating zones of the $i$ th generator consist of $M_{i}+1$ disjoint regions:

$$
\left\{\begin{array}{l}
\underline{P}_{i} \leqslant P_{i} \leqslant \underline{P}_{i}^{1}, \text { or } \\
\bar{P}_{i}^{j} \leqslant P_{i} \leqslant \underline{P}_{i}^{j+1}, \text { for } j=1, \ldots, M_{i}-1, \text { or } \\
\bar{P}_{i}^{M_{i}} \leqslant P_{i} \leqslant \bar{P}_{i} .
\end{array}\right.
$$

The prohibited operating zones are mainly due to the faults in the machines themselves or the associated auxiliary devices, e.g., boilers. These faults tend to cause instability of generators in some regions of outputs. The prohibited operating zones lead to the discontinuity (non-convexity) of the feasible region of the EDP, rendering the EDP non-convex.

Let us revisit the problem (33). The first derivative of $L_{i}^{o}$ is given by

$$
\frac{d L_{i}^{o}}{d P_{i}}=f_{i}\left(P_{i}\right)-\frac{\lambda}{p f_{i}[k]} .
$$

If there are no inequality constraints, the optimal solution to (33), denoted by $P_{i}^{o}$, is such that $\partial L^{o} / \partial P_{i}=0$, i.e.,

$$
P_{i}^{o}=g_{i}\left(\frac{\lambda}{p f_{i}[k]}\right)=h_{i}(\lambda)
$$

where $g_{i}(\cdot)$ is the inverse function of $f_{i}(\cdot)$. If $P_{i}^{o}$ happens to belong to the set $\Omega_{i}$, then $P_{i}^{o}$ minimizes $L_{i}^{o}$ with respect to not only the set $\Omega_{i}$, but also the whole domain of real numbers. If $P_{i}^{o}$ does not belong to $\Omega_{i}$, there are 3 cases:

1) $P_{i}^{o}>\bar{P}_{i}$ : From the assumption 1, it follows that $f_{i}\left(P_{i}\right)$ is continuously increasing in $\mathbb{R}_{+}$, therefore $d L_{i}^{o} / d P_{i}$ is also continuously increasing in $\mathbb{R}_{+}$. Since $P_{i}^{o}>\bar{P}_{i}$ and $d L_{i}^{o} / d P_{i}\left(P_{i}^{o}\right)=0$, it follows that $d L_{i}^{o} / d P_{i}\left(P_{i}\right)<0$. Therefore $L_{i}^{o}$ is monotonically decreasing in $\Omega_{i}$ and $P_{i}^{\star}=\bar{P}_{i}$ is the minimizer of $L_{i}^{o}$

2) $P_{i}^{o}<\underline{P}_{i}$ : This case can also be analyzed using the above technique. Similarly, we have $P_{i}^{\star}=\underline{P}_{i}$. 
3) $\underline{P}_{i}^{j}<P_{i}^{o}<\bar{P}_{i}^{j}$ : In this case $P_{i}^{o}$ falls into one of the prohibited operating zones. Since $d L_{i}^{o} / d P_{i}\left(P_{i}^{o}\right)=0$, we conclude that for any $P_{i} \in \Omega_{i}$,

$$
\begin{cases}L_{i}^{o}\left(P_{i}\right) \geqslant L_{i}^{o}\left(\underline{P}_{i}^{j}\right), & \text { for } P_{i}<P_{i}^{o} \\ L_{i}^{o}\left(P_{i}\right) \geqslant L_{i}^{o}\left(\bar{P}_{i}^{j}\right), & \text { for } P_{i}>P_{i}^{o}\end{cases}
$$

Therefore the optimal solution is either $\underline{P}_{i}^{j}$ or $\bar{P}_{i}^{j}$. We then define

$$
\begin{gathered}
\lambda_{i}^{j}=\frac{F_{i}\left(\bar{P}_{i}^{j}\right)-F_{i}\left(\underline{P}_{i}^{j}\right)}{\bar{P}_{i}^{j}-\underline{P}_{i}^{j}}, \\
L_{i}^{o}\left(\bar{P}_{i}^{j}\right)-L_{i}^{o}\left(\underline{P}_{i}^{j}\right)=F_{i}\left(\bar{P}_{i}^{j}\right)-F_{i}\left(\underline{P}_{i}^{j}\right)-\frac{\lambda\left(\bar{P}_{i}^{j}-\underline{P}_{i}^{j}\right)}{p f_{i}[k]} .
\end{gathered}
$$

It follows that

- If $L_{i}^{o}\left(\bar{P}_{i}^{j}\right)-L_{i}^{o}\left(\underline{P}_{i}^{j}\right)>0$, then $\lambda<p f_{i}[k] \lambda_{i}^{j}$ and $P_{i}^{\star}=\underline{P}_{i}^{j}$;

- If $L_{i}^{o}\left(\bar{P}_{i}^{j}\right)-L_{i}^{o}\left(\underline{P}_{i}^{j}\right)<0$, then $\lambda>p f_{i}[k] \lambda_{i}^{j}$ and $P_{i}^{\star}=\bar{P}_{i}^{j}$;

- If $L_{i}^{o}\left(\bar{P}_{i}^{j}\right)-L_{i}^{o}\left(\underline{P}_{i}^{j}\right)=0$, then $\lambda=p f_{i}[k] \lambda_{i}^{j}$ and $P_{i}^{\star}=\underline{P}_{i}^{j}$ or $\bar{P}_{i}^{j}$.

According to the above analysis, replace the mapping (28) with the following one in the case of prohibited operating zones:

$$
\mathcal{P}_{i}(\lambda)= \begin{cases}\underline{P}_{i}, & h_{i}(\lambda)<\underline{P}_{i}, \\ h_{i}(\lambda), & h_{i}(\lambda) \in \Omega_{i}, \\ \underline{P}_{i}^{j}, & \underline{P}_{i}^{j}<h_{i}(\lambda)<\bar{P}_{i}^{j}, \lambda<p f_{i}[k] \lambda_{i}^{j}, \\ \bar{P}_{i}^{j}, & \underline{P}_{i}^{j}<h_{i}(\lambda)<\bar{P}_{i}^{j}, \lambda \geqslant p f_{i}[k] \lambda_{i}^{j}, \\ \bar{P}_{i}, & h_{i}(\lambda)>\bar{P}_{i} .\end{cases}
$$

\section{Numerical Simulations}

In this section, we present three case studies to fully demonstrate the performance of the proposed algorithm, which are tested on IEEE 30-bus system, IEEE 14-bus system, and 15-unit system, respectively. 


\subsection{Case1: IEEE 30-bus System}

In this case, we apply our distributed algorithm proposed in Section 4 to the lossy EDP on the IEEE 30-bus system [26]. Quadratic cost functions and transmission losses are considered, while prohibited operating zones are neglected, which implies that the conventional lambda-iteration method [15] can also solve this case. The generation cost coefficients are adopted from [10], and the $\mathbf{B}$ coefficients for the IEEE 30-bus system are adopted from $[27]$.

In this case the total power demand is $P_{L}=300 \mathrm{MW}$. We first give the results of this case neglecting the transmission losses. The optimal Lagrange multiplier $\lambda^{*}=6.5944 \mathrm{MU} / \mathrm{MWh}$, and the optimal power assignments are $P_{1}^{*}=57.43 \mathrm{MW}, P_{2}^{*}=59.91 \mathrm{MW}, P_{3}^{*}=37.06 \mathrm{MW}, P_{4}^{*}=43.24 \mathrm{MW}$, $P_{5}^{*}=51.18 \mathrm{MW}, P_{6}^{*}=51.18 \mathrm{MW}$, where MU is short for monetary unit.

We then show the results of the lossy EDP using the proposed algorithm. We set $\lambda^{+}(0)=10 \mathrm{MU} / \mathrm{MWh}$ and $\lambda^{-}(0)=0 \mathrm{MU} / \mathrm{MWh}$, which are sufficient to ensure $\lambda^{*} \in\left[\lambda^{-}(0), \lambda^{+}(0)\right]$. In the calculation of $P_{i}[k]$ 's and $\lambda[k]$, we artificially set the bisection number to 15 , such that for each $k, \mid \lambda(15)-$ $\lambda[k]\left|\leqslant \frac{1}{2}\right| \lambda^{+}(14)-\lambda^{-}(14)\left|=\frac{1}{2^{15}}\right| \lambda^{+}(0)-\lambda^{-}(0) \mid \approx 0.0003$.

The updates of $\lambda[k]$ and $P_{i}[k]$ 's are shown in Fig. 1 and Fig. 2, respectively. We set the iteration step to 10 , while the convergence is already reached at $k=7$. It can be seen from Fig. 1 that both the proposed algorithm and the conventional lambda-iteration method converge to optima, as the two lambda curves coincides. The optimal Lagrange multiplier $\lambda^{*}=6.8600 \mathrm{MU} / \mathrm{MWh}$, and the optimal power assignments are $P_{1}^{*}=52.36 \mathrm{MW}, P_{2}^{*}=60.05 \mathrm{MW}$, $P_{3}^{*}=41.38 \mathrm{MW}, P_{4}^{*}=45.99 \mathrm{MW}, P_{5}^{*}=53.44 \mathrm{MW}, P_{6}^{*}=51.88 \mathrm{MW}$. The aggregate generation output is $\sum_{i=1}^{n} P_{i}^{*}=305.11>300 \mathrm{MW}$, which is caused by the transmission losses. Furthermore, the presence of transmission losses slightly increase the optimal incremental cost (Lagrange multiplier) by $6.8600-6.5944=0.2656 \mathrm{MU} / \mathrm{MWh}$.

\subsection{Case 2: IEEE 14-bus System}

It may seems doubtful to the reader that Case 2 is downsized to IEEE 14-bus system, while Case 1 is already tested on 30-bus system. The reason is that we have observed the the oscillating problem of conventional lambda iterations for the 14-bus system. Therefore, this case can help test the performance of the damping mechanism.

We replace the 3 synchronous condensers with 3 generators, so there are 5 generators in this system, whose parameters are adopted from [13]. The $\mathbf{B}$ 


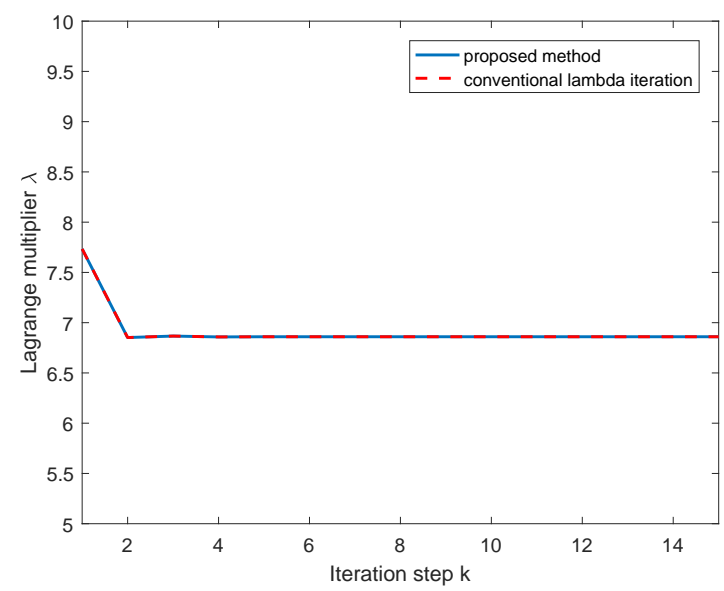

Figure 1: Iteration results of the Lagrange multiplier $\lambda[k]$ in Case 1.

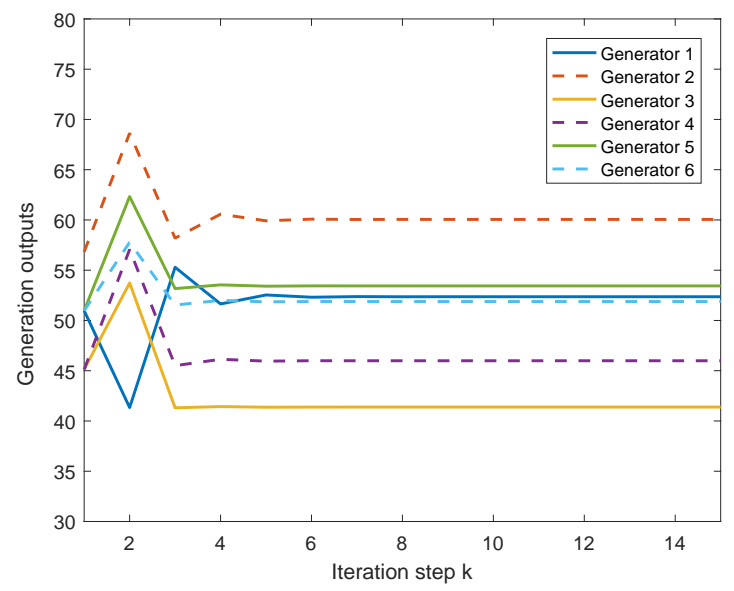

Figure 2: Iteration results of the generators' outputs $P_{i}[k]$ in Case 1.

coefficients for the IEEE 14-bus system are calculated from [28]. In this case the total power demand is $P^{\text {load }}=250 \mathrm{MW}$. We use the same computational setups as Case 1.

The evolutions of $\lambda[k]$ and $P_{i}[k]$ 's are shown in Fig. 3 and Fig. 4, respectively. From Fig. 3, we can see that the conventional lambda-iteration method exhibits the oscillatory behavior, i.e., after about 5 iterations, $\lambda[k]$ oscillates between 7.0316 MU/MWh and 7.1545 MU/MWh, while the pro- 


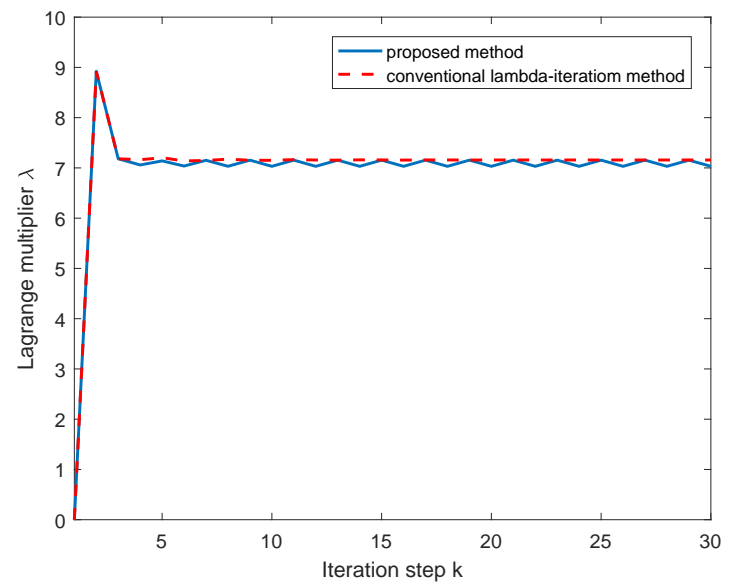

Figure 3: Iteration results of the Lagrange multiplier $\lambda[k]$ in Case 2 without prohibited operating zones.

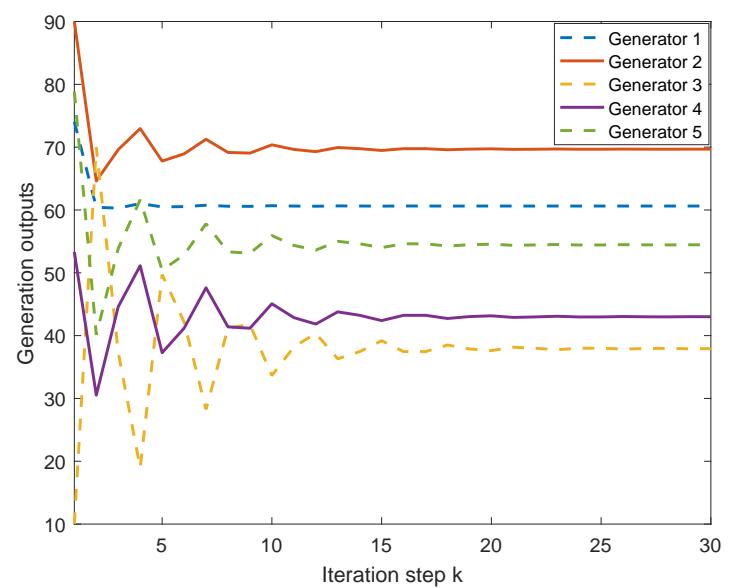

Figure 4: Iteration results of the generators' outputs $P_{i}[k]$ in Case 2 without prohibited operating zones.

posed algorithm converges to $7.1577 \mathrm{MU} / \mathrm{MWh}$ due to the damping operator. The aggregate generation output is $\sum_{i=1}^{n} P_{i}^{*}=265.69>250 \mathrm{MW}$, which is caused by the transmission losses.

In the IEEE 14-bus case, we further test the proposed algorithm's performance in the presence of prohibited operating zones. We impose prohibited 


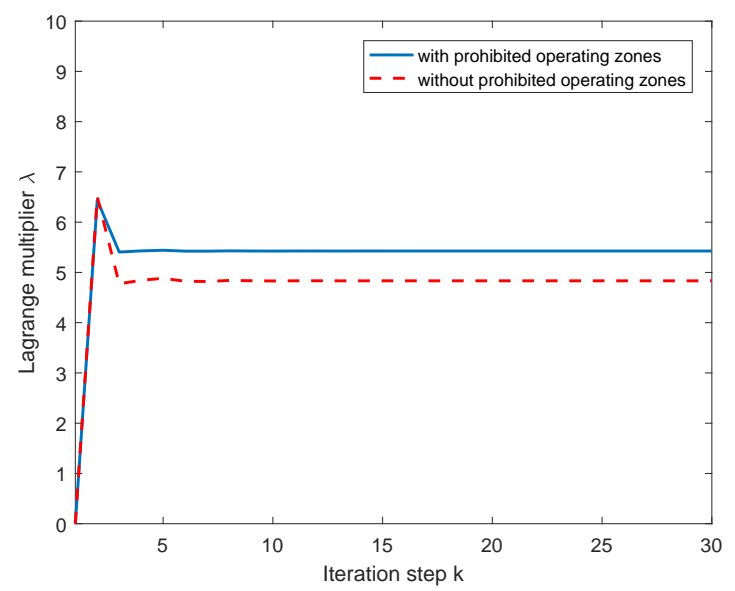

Figure 5: Iteration results of the Lagrange multiplier $\lambda[k]$ in Case 2 with prohibited operating zones.

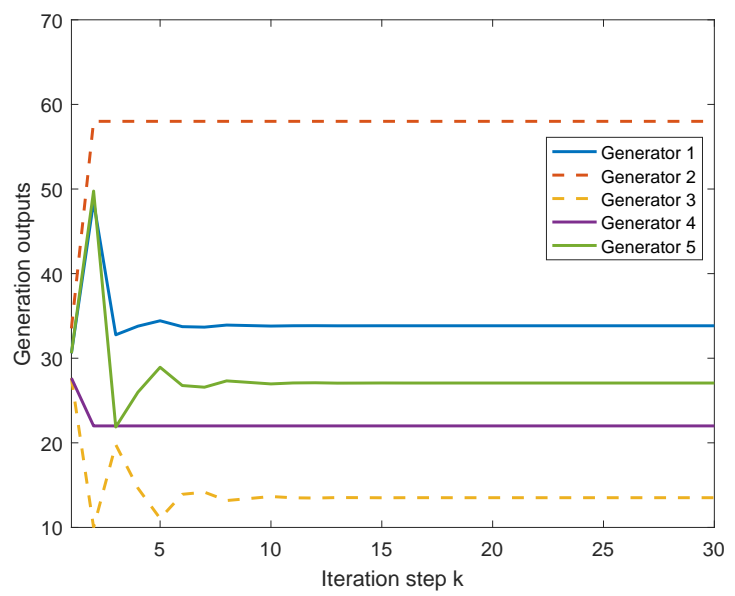

Figure 6: Iteration results of the generators' outputs $P_{i}[k]$ in Case 2 with prohibited operating zones.

operating zones of $20 \sim 40 \%$ and $60 \sim 80 \%$ of each generator's capacity range. To better illustrate the impact of the prohibited operating zones, we set the total demand $P^{\text {load }}=150 \mathrm{MW}$.

The evolutions of $\lambda[k]$ and $P_{i}[k]$ 's are shown in Fig. 5 and 6 , respectively. One counter-intuitive finding from Fig. 5 is that the optimal Lagrange mul- 
tiplier $\lambda^{*}=4.833 \mathrm{MU} / \mathrm{MWh}$ with the prohibited operating zones is lower than $\lambda^{*^{\prime}}=5.426 \mathrm{MU} / \mathrm{MWh}$ without the prohibited operating zones. Intuitively, due to the existence of prohibited operating zones, the optimal power assignments are forced away from those without prohibited operating zones, probably driving the marginal cost up. But on the other hand, the optimal aggregated cost with the prohibited zones is $F^{*}=648.33 \mathrm{MU} / \mathrm{h}$, which is larger than $F^{*^{\prime}}=631.81 \mathrm{MU} / \mathrm{h}$ without the prohibited operating zones. For generators 2 and 4, their optimal power assignments are $58 \mathrm{MW}$ and $22 \mathrm{MW}$, which take the boundary value of their prohibited operating zones, while in the absence of prohibited operating zones their optimal assignment are 40.14 MW and 20.09 MW, respectively.

\subsection{Case 3: 15-unit System}

Case 1 and Case 2 consider the economic dispatch of 6 and 5 energy resources, respectively, which is relatively small-scale. In this case we apply the overall method to the 15-unit system to test the applicability to large systems. The system parameters are adopted from [29]. Specifically, the total installed capacity is $3452 \mathrm{MW}$, and the total power demand is $2630 \mathrm{MW}$.

We first show the results without considering prohibited operating zones, as illustrated in Fig. 7-9. It can be seen from Fig. 7 and 8 that the conventional lambda iteration has suffered oscillatory problem. Although the oscillations seem to happen between two points, in fact 3 point with two of them having subtle difference are involved. Therefore, in this case we adopt the damping operator

$$
D[k]=\frac{P[k]+\ldots+P[k-l+1]}{l}
$$

with $l=3$ to successfully suppress the oscillations.

Finally, we take into consideration the prohibited operating zones for the 15-unit case. Similarly, the damping mechanism has successfully suppressed the oscillations and the lossy EDP has been optimally solved, as shown in Fig. 10 and 11.

\section{Concluding Remarks}

This paper proposed a consensus-based lambda-iteration algorithm to tackle a more practical economic dispatch problem involving general cost

functions, transmission losses, and prohibited operating zones. The proposed 


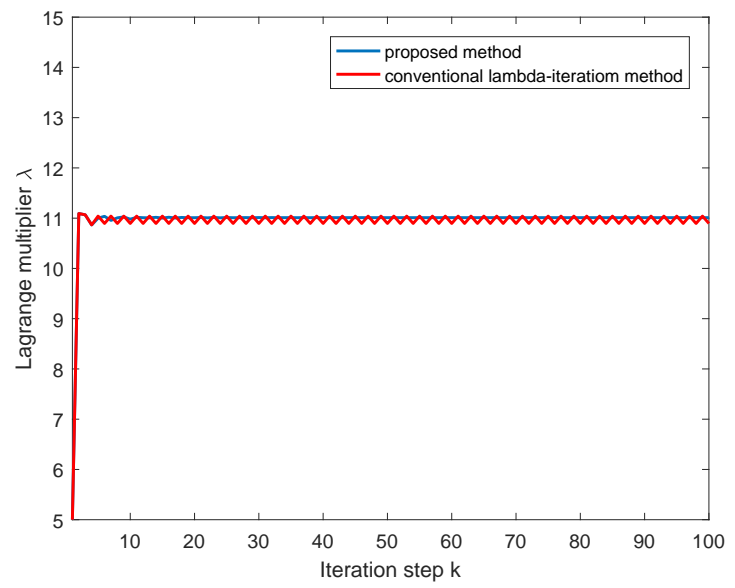

Figure 7: Iteration results of the Lagrange multiplier $\lambda[k]$ in Case 3 without prohibited operating zones.

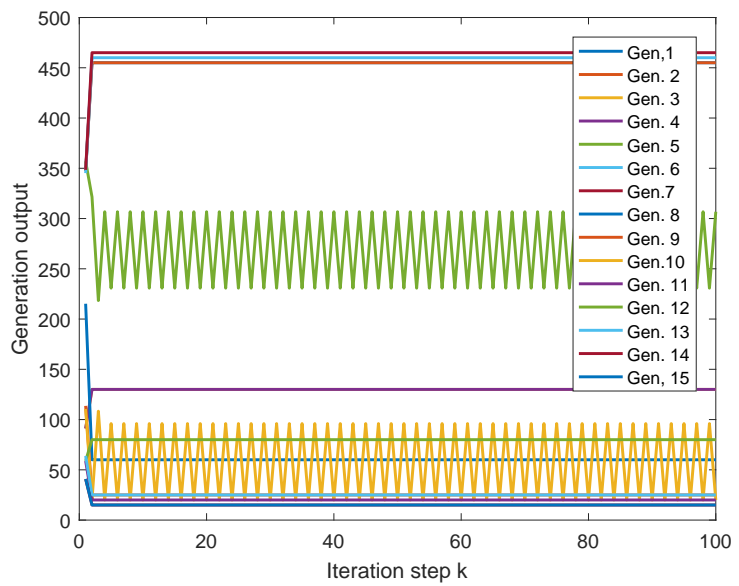

Figure 8: Generation outputs of conventional lambda iterations in Case 3 without prohibited operating zones.

method outperforms the conventional one by effectively dealing with the prohibited operating zones and avoiding the oscillatory behavior. Moreover, the proposed algorithm is distributed in the sense that computation is conducted by the nodes locally and information is merely exchanged with neighbors. Although using only local communication and distributed computation, the 


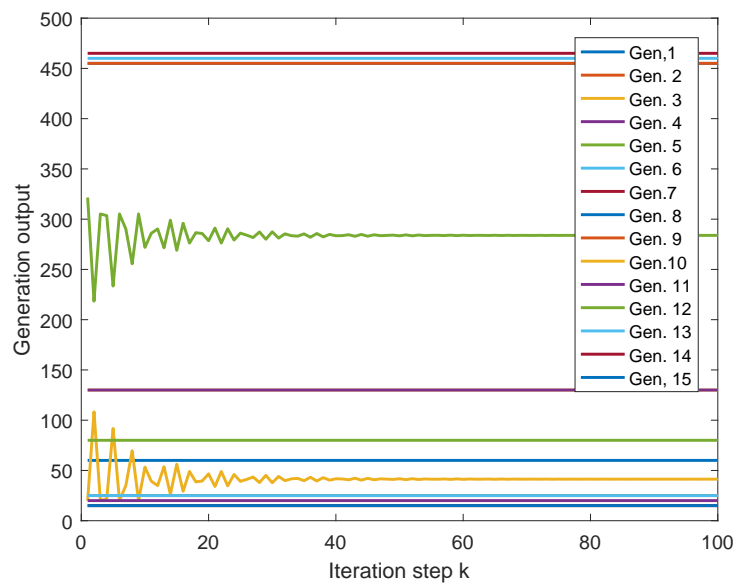

Figure 9: Generation outputs of proposed method in Case 3 without prohibited operating zones.

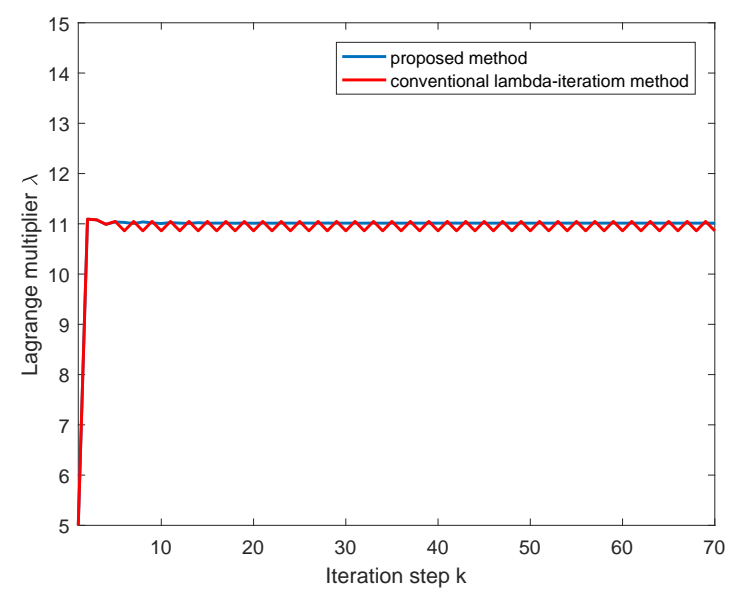

Figure 10: Iteration results of the Lagrange multiplier $\lambda[k]$ in Case 3 with prohibited operating zones.

proposed approach still achieves exactly the same optimality as the traditional methods based on global information and central computation. Extensive case studies have been presented to fully show the performance of the proposed algorithm. As future work, more constraints will be considered, such as transmission line capacity limit and spinning reserves. 


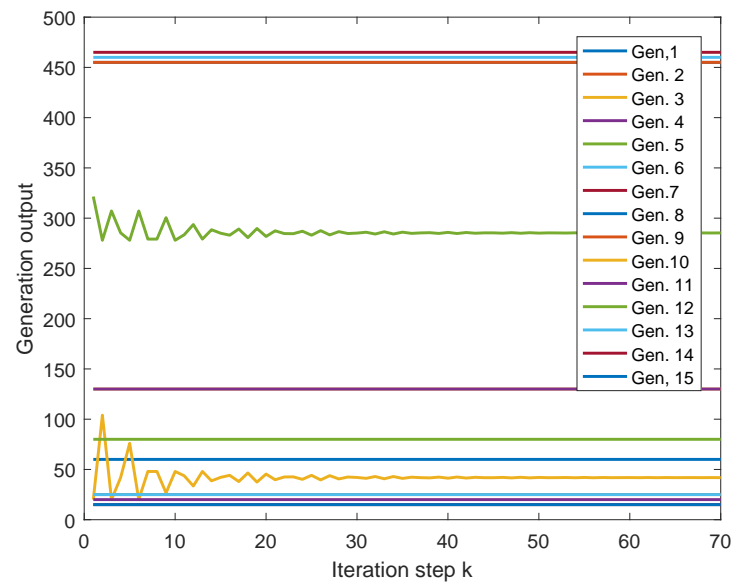

Figure 11: Generation outputs of proposed method in Case 3 with prohibited operating zones.

\section{Acknowledgements}

This work was supported by the National Natural Science Foundation of China (grant No. U1766201).

\section{References}

[1] G. B. Gharehpetian, S. M. M. Agah, Distributed Generation Systems: Design, Operation and Grid Integration, Butterworth-Heinemann, 2017.

[2] J. Ansari, A. Gholami, A. Kazemi, Multi-agent systems for reactive power control in smart grids, International Journal of Electrical Power \& Energy Systems 83 (2016) $411-425$.

[3] F. Rezaei, S. Esmaeili, Decentralized reactive power control of distributed PV and wind power generation units using an optimized fuzzy-based method, International Journal of Electrical Power \& Energy Systems 87 (2017) $27-42$.

[4] A. M. Ospina, N. Quijano, Distributed control of small-scale power systems using noncooperative games, International Journal of Electrical Power \& Energy Systems 82 (2016) 535 - 544. 
[5] Y. Sun, C. Zhong, X. Hou, J. Yang, H. Han, J. M. Guerrero, Distributed cooperative synchronization strategy for multi-bus microgrids, International Journal of Electrical Power \& Energy Systems 86 (2017) 18 28.

[6] T. Sui, K. You, M. Fu, D. Marelli, Stability of mmse state estimators over lossy networks using linear coding, Automatica 51 (C) (2015) 167174 .

[7] T. Sui, D. E. Marelli, M. Fu, R. Lu, Accuracy analysis for distributed weighted least-squares estimation in finite steps and loopy networks, Automatica 97 (2018) 82-91.

[8] Z. Zhang, X. Ying, M.-Y. Chow, Decentralizing the economic dispatch problem using a two-level incremental cost consensus algorithm in a smart grid environment, in: Proc. IEEE North American Power Symposium (NAPS), Boston, MA, 2011, pp. 1-7.

[9] Z. Zhang, M.-Y. Chow, Incremental cost consensus algorithm in a smart grid environment, in: Proc. IEEE Power and Energy Society General Meeting, San Diego, CA, 2011, pp. 1-6.

[10] S. Kar, G. Hug, Distributed robust economic dispatch in power systems: A consensus + innovations approach, in: Proc. IEEE Power and Energy Society General Meeting, San Diego, CA, 2012, pp. 1-8.

[11] S. Yang, S. Tan, J. X. Xu, Consensus based approach for economic dispatch problem in a smart grid, IEEE Trans. Power Syst. 28 (4) (2013) 4416-4426.

[12] H. Xing, Y. Mou, M. Fu, Z. Lin, Consensus based bisection approach for economic power dispatch, in: Proc. IEEE Conference on Decision and Control, 2014, pp. 3789-3794.

[13] H. Xing, Y. Mou, M. Fu, Z. Lin, Distributed bisection method for economic power dispatch in smart grid, IEEE Trans. Power Syst. 30 (6) (2015) 3024-3035.

[14] H. Xing, Z. Lin, M. Fu, B. F. Hobbs, Distributed algorithm for dynamic economic power dispatch with energy storage in smart grids, Iet Control Theory \& Applications 11 (11) (2017) 1813-1821. 
[15] A. J. Wood, B. F. Wollenberg, Power Generation, Operation, and Control, John Wiley \& Sons, 1996.

[16] H. Happ, Optimal power dispatch: A comprehensive survey, IEEE Trans. Power App. Syst. 96 (3) (1977) 841-854.

[17] P. Chen, H. Chang, Large-scale economic dispatch by genetic algorithm, IEEE Trans. Power Syst. 10 (4) (1995) 1919-1926.

[18] C. Chen, N. Chen, Direct search method for solving economic dispatch problem considering transmission capacity constraints, IEEE Trans. Power Syst. 16 (4) (2001) 764-769.

[19] G. Binetti, A. Davoudi, F. L. Lewis, D. Naso, B. Turchiano, Distributed consensus-based economic dispatch with transmission losses, IEEE Trans. Power Syst. 29 (4) (2014) 1711-1720.

[20] F. N. Lee, A. M. Breipohl, Reserve constrained economic dispatch with prohibited operating zones, IEEE Trans. Power Syst. 8 (1) (1993) 246254 .

[21] J. Fan, J. McDonald, A practical approach to real time economic dispatch considering unit's prohibited operating zones, IEEE Trans. Power Syst. 9 (4) (1994) 1737-1743.

[22] A. Bemporad, M. Heemels, M. Johansson, Networked Control Systems, Vol. 406, Springer, 2010.

[23] A. D. Dominguez-Garcia, S. T. Cady, C. N. Hadjicostis, Decentralized optimal dispatch of distributed energy resources, in: Proc. IEEE Conference on Decision and Control, Maui, Hawaii, 2012, pp. 3688-3693.

[24] W. T. Elsayed, E. F. El-Saadany, A fully decentralized approach for solving the economic dispatch problem, IEEE Trans. Power Syst. 30 (4) (2015) 2179-2189.

[25] J. J. Grainger, W. D. Stevenson, Power System Analysis, Vol. 621, McGraw-Hill New York, 1994.

[26] Power system test case archive.

URL http://www. ee.washington.edu/research/pstca/ 
[27] D. Gong, Y. Zhang, C. Qi, Environmental/economic power dispatch using a hybrid multi-objective optimization algorithm, International Journal of Electrical Power \& Energy Systems 32 (6) (2010) 607-614.

[28] M. A. Pai, D. Chatterjee, Computer techniques in power system analysis, McGraw-Hill Education (India), 2014.

[29] Z.-L. Gaing, Particle swarm optimization to solving the economic dispatch considering the generator constraints, IEEE Trans. Power Syst. 18 (3) (2003) 1187-1195. 